Journal of Fundamental and Applied Sciences

ISSN 1112-9867

Available online at

http://www.jfas.info

\title{
SPECTROSCOPIC DIAGNOSIS IN ELECTRONIC TEMPERATURE OF PHOTOIONISE PLASMAS
}

\author{
A. K. Ferouani ${ }^{*}$ and M. K. Inal \\ Université Abou Bekr Belkaïd Tlemcen, Faculté des Sciences, Département de \\ Physique, Laboratoire de Physique Théorique, B.P 119 Tlemcen 13 000, Algérie
}

Received: 07 June 2009 / Accepted: 27 October 2009 / Published online: 31 December 2009

\begin{abstract}
In this work, we are interested in the diagnostics in electronic temperature of a plasma purely photoionized, based on the intensity ration of lines emitted by ions helium-like, which have an atomic number $Z$ relatively small. We considered the three lines corresponding to the transitions starting from the excited levels $1 \mathrm{~s} 2 l$ towards the fundamental level $1 \mathrm{~s}^{2}{ }^{1} \mathrm{~S}_{0}$, like appropriate lines. More precisely, the line of resonance $\mathrm{w}$ due to the transition $1 \mathrm{~s} 2 \mathrm{p}{ }^{1} \mathrm{P}_{1} \rightarrow 1 \mathrm{~s}^{2}{ }^{1} \mathrm{~S}_{0}$, the line of intercombinaison $(\mathrm{x}, \mathrm{y})$ $1 \mathrm{~s} 2 \mathrm{p}^{3} \mathrm{P}_{2,1} \rightarrow 1 \mathrm{~s}^{2}{ }^{1} \mathrm{~S}_{0}$ as well as prohibited line $\mathrm{z}$ due to the transition $1 \mathrm{~s}^{2}{ }^{3} \mathrm{~S}_{1} \rightarrow 1 \mathrm{~s} 2{ }^{1}$ $\mathrm{S}_{0}$. These lines appear clearly in the spectra of astrophysical plasmas. As helium-like ion, we chose two, the oxygen $\mathrm{O}^{6+}(\mathrm{Z}=8)$ and neon $\mathrm{Ne}^{8+}(\mathrm{Z}=10)$. We carried out calculations of the ration of lines intensity $\mathrm{G}=(\mathrm{z}+\mathrm{x}+\mathrm{y}) / \mathrm{w}$ of $\mathrm{O}^{6+}$ and $\mathrm{Ne}^{8+}$ according to the electronic temperature in the range going from $10^{5}$ to $10^{7} \mathrm{~K}$. We will see that, like it was shown by Gabriel and Jordan in 1969 [1], this intensity ration can be very sensitive to the temperature electronic and practically independent of the electronic density. Consequently, the ration $\mathrm{G}$ can be used to determine in a reliable way the electronic temperature of plasma observed [2].
\end{abstract}

Key words: Strongly charged ions, lines emission, radiative and dielectronic recombination.

Author Correspondence, e-mail: ferouani_karim@yahoo.fr

ICID: 1040442 


\section{1- INTRODUCTION}

Souvent appelé le quatrième état de la matière, le plasma se rencontre en quantité abondante, en fait bien plus abondante que n'importe quel autre état de la matière, dans la nature que ce soit dans les étoiles, la magnétosphère ou autre environnement planétaire. Il peut être créé par divers moyens en laboratoire. Les principales méthodes de diagnostic des plasmas chauds sont des méthodes faisant appel au rayonnement électromagnétique émis par ces plasmas [3]. L'analyse spectroscopique du rayonnement sur l'ensemble du spectre électromagnétique est interprétée au moyen de modèles théoriques qui expliquent les mécanismes de production et d'émission du rayonnement. L'étude de ce rayonnement permet de déterminer la nature des ions, leurs répartitions et leurs niveaux d'énergie. En outre, les intensités relatives de certaines raies émises par les ions du plasma fournissent de précieux renseignements sur les conditions physiques qui règnent dans le plasma à savoir la densité et la température électronique, l'état d'ionisation, etc. Ces méthodes spectroscopiques ont été appliquées comme outil efficace de diagnostic des plasmas aussi bien astrophysiques que ceux produits en laboratoires à des fins de fusions par confinement magnétiques ou inertiel et également dans la réalisation des lasers $X[4][5]$.

Dans les plasmas astrophysiques exposés à des sources avoisinantes intenses de rayonnement $X$, la photoionisation peut constituer un mécanisme important pour la production des ions hautement chargés. De tels plasmas font l'objet, depuis ces quelques dernières années, d'études intensives et minitieuses par les astrophysiciens. Les raies d'émission à partir de ces plasmas sont formées principalement par les processus de recombinaisons radiative et diélectronique [6]. Nous nous sommes intéressé dans ce travail au diagnostic spectroscopique en température électronique $\mathrm{G}(\mathrm{Te})=(\mathrm{z}+\mathrm{x}+\mathrm{y}) / \mathrm{w}$ des plasmas photoionisés en se basant sur le rapport d'intensité de raies émises par les ions héliumoïdes multichargés.

Les ions héliumoïdes (He-like) sont très abondants dans les plasmas du fait de la stabilité de la structure en couche fermée de leur niveau fondamental $1 \mathrm{~s}^{2}{ }^{1} \mathrm{~S}_{0}$, ce qui nécessite une quantité d'énergie relativement grande pour qu'ils soient ionisés ou même excités. Les trois principales raies en émission (domaine des rayons $\mathrm{X}$ ) de ces ions sont les: la raie de résonance $\mathrm{w}: 1 \mathrm{~s} 2 \mathrm{p}{ }^{1} \mathrm{P}_{1} \rightarrow 1 \mathrm{~s}^{2}{ }^{1} \mathrm{~S}_{0}$, la raie interdite $\mathrm{z}: 1 \mathrm{~s} 2 \mathrm{~s}{ }^{3} \mathrm{~S}_{1} \rightarrow 1 \mathrm{~s}^{2} \mathrm{~S}_{0}$ et les raies d'intercombinaisons (x,y): $1 \mathrm{~s} 2 \mathrm{p}^{3} \mathrm{P}_{2,1} \rightarrow 1 \mathrm{~s}^{2}{ }^{1} \mathrm{~S}_{0}$, respectivement). Toutes ces raies correspondent aux transitions de la couche $n=2$ vers la couche $n=1$ (niveau 
fondamental) (Figure 1). Ces trois raies sont depuis longtemps largement utilisées comme diagnostics de densité [7]-[8] et de température électronique pour le soleil et ses éruptions et quelques fois pour les restes de Supernovae et le milieu interstellaire, i.e. pour les plasmas collisionnels [9]. En effet, Gabriel et Jordan [1]-[5] avaient montré que le rapport de leur intensité défini ci-dessous est sensible à la température électronique (rapport appelé $\mathrm{G}(\mathrm{Te}))$ :

$$
G(T e)=\frac{z+(x+y)}{w}
$$

Ces diagnostics ont l'avantage que les raies sont émises par un même ion, ce qui leur permet d'être indépendants de l'abondance des éléments, et par conséquent d'éliminer un paramètre libre lors des diagnostics. Du point de vue observationnel, la séparation de ces trois raies a été récemment rendu possible dans le cas des plasmas non solaires grâce aux satellites X (Chandra, XMM, Astro-E). Ces types de diagnostics pourront être utilisés non seulement pour les plasmas collisionnels mais également pour les plasmas photoionisés (Pradhan (1985) [2], Liedahl (1999) [10] et Porquet et al. (2000)[11]).

\section{Intensité d'une raie spectrale d'émission}

L'intensité d'une raie spectrale due à la transition d'un niveau supérieur $j$ vers un niveau inférieur $i$ dans un atome (ou ion) $X$ est donnée par la relation suivants :

$$
I(j \rightarrow i)=N_{j} A(j \rightarrow i) h v
$$

Où $N_{j}$ désigne la densité des atomes $\mathrm{X}$ dans le niveau supérieur $j\left(\mathrm{en}^{-3}\right), A(j \rightarrow i)$ est la probabilité de transition radiative spontanée de $j$ vers $i\left(\mathrm{en} \mathrm{s}^{-1}\right)$ et hv est l'énergie du photon émis lors de la transition (en eV).

En substituant maintenant l'équation (2) dans l'équation (1), on peut écrire comme équation :

$$
\begin{gathered}
G(T e)=\frac{I\left(2{ }^{3} S_{1} \rightarrow 1{ }^{1} S_{0}\right)+\left[I\left(2{ }^{3} P_{1} \rightarrow 1{ }^{1} S_{0}\right)+I\left(2{ }^{3} P_{2} \rightarrow 1{ }^{1} S_{0}\right)\right]}{I\left(2{ }^{1} P_{1} \rightarrow 1{ }^{1} S_{0}\right)} \\
G(T e)=\frac{N_{2} A_{21} h v_{z}+\left(N_{4} A_{41} h v_{x}+N_{5} A_{51} h v_{y}\right)}{N_{4} A_{71} h v_{w}}=\frac{N_{2} A_{21}+\left(N_{4} A_{41}+N_{5} A_{51}\right)}{N_{7} A_{71}}
\end{gathered}
$$

Cette expression peut être approximée par :

$$
G\left(T_{e}\right)=\frac{N_{2} A_{21}+N_{4} A_{41}}{N_{7} A_{71}}
$$


En utilisant le fait que $\mathrm{A}_{51}<<\mathrm{A}_{52}, \mathrm{~A}_{42}<<\mathrm{A}_{41}$ et $\mathrm{h} v_{\mathrm{z}} \approx \mathrm{h} v_{\mathrm{x}} \approx \mathrm{h} v_{\mathrm{y}} \approx \mathrm{h} v_{\mathrm{w}}$. En effet, pour des ions de $Z$ petit le niveau 5 se dépeuple par émission spontanée essentiellement vers le niveau 2 ; par exemple dans le cas de l'ion $\mathrm{Ne}^{8+}$, le rapport de branchement pour la transition radiative de 5 vers $2, \mathrm{~A}_{52} /\left(\mathrm{A}_{51}+\mathrm{A}_{52}\right)$, vaut 0,98 , comme il sera vu ultérieurement.

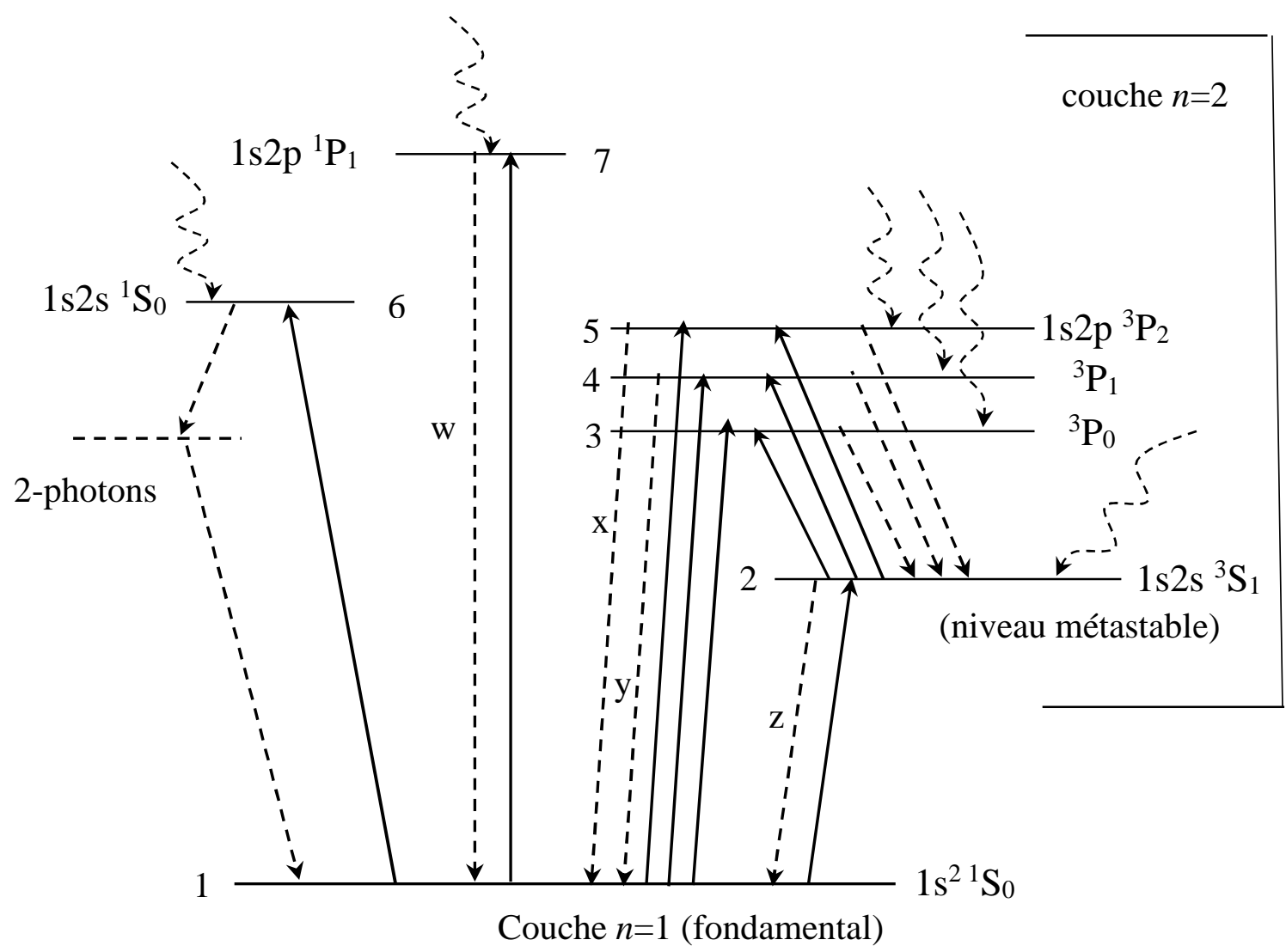

Fig.1. Diagramme de Gotrian des ions héliumoïdes limité aux couches $n=1$ et $n=2$. Trait en continu: excitation collisionnelle, trait en discontinu: désexcitation radiative, tirets courbés: recombinaisons radiative et diélectronique. Ce diagramme n'est pas à l'échelle, en effet, la différence d'énergie entre les niveaux excités de la couche $\mathrm{n}=2$ et le niveau fondamental (couche $\mathrm{n}=1$ ) est très grand par rapport à celle entre les niveaux excités de la couche $n=2$.

Dans ce travail, il y a deux types de plasmas doivent être étudiés. Par la suite, je référerai comme plasma "purement photoionisé" un plasma où la photoionisation est le processus d'ionisation dominant (source d'ionisation externe au plasma). Pour un tel plasma, les recombinaisons des ions hydrogénoïdes (H-like) vers les ions héliumoïdes 
(He-like) sont dominantes par rapport aux excitations collisionnelles électroniques à partir du niveau fondamental des ions héliumoïdes. En effet, les différences d'énergie entre le niveau fondamental de la couche $n=1$ et les niveaux excités de la couche $n=2$ sont très grands et nécessitent une très grande température pour permettre la transition d'un électron. Ce qui explique que les raies sont principalement formées par recombinaison.

Un plasma "hybride" signifiera un plasma partiellement photoionisé, i.e. avec un processus supplémentaire d'ionisation, ici collisionnel (source interne d'ionisation). Dans ce type de plasma, les processus d'excitation collisionnelle à partir du niveau fondamental peuvent être aussi importants, sinon plus, que les recombinaisons des ions hydrogénoïdes. Dans ce cas la température est assez élevée pour permettre le passage d'un électron de la couche $n=1$ à la couche $n=2$ et les raies sont formées par l'action combinée de l'excitation collisionnelle et de la recombinaison.

Dans le cas d'un plasma photoionisé pur, la recombinaison radiative est le processus dominant aux basses températures alors que la recombinaison diélectronique est dominante aux hautes températures. Les excitations collisionnelles à l'intérieur des niveaux de la couche $n=2$ peuvent être également très importantes lorsque la densité des électrons est assez grande. En effet, la différence d'énergie entre les niveaux de la couche $n=2$ est très petite et par conséquent une température même modérée caractéristique des plasmas photoionisés est suffisante pour exciter un électron du niveau métastable $1 \mathrm{~s} 2 \mathrm{~s}{ }^{3} \mathrm{~S}_{1}$ vers l'un des niveaux du terme $1 \mathrm{~s} 2 \mathrm{p}{ }^{3} \mathrm{P}$. Le transfert collisionnel de population de $1 \mathrm{~s} 2 \mathrm{~s}{ }^{3} \mathrm{~S}_{1}$ vers $1 \mathrm{~s} 2 \mathrm{p}{ }^{3} \mathrm{P}_{0,1,2} \mathrm{n}$ 'est, en fait, efficace que si la densité des électrons de collision est assez élevée pour contrecarrer la désexcitation radiative spontanée de $1 \mathrm{~s} 2 \mathrm{~s}{ }^{3} \mathrm{~S}_{1}$.

\section{Diagnostic en température électronique des plasmas photoionises}

Nous introduisons le paramètre $\mathrm{X}_{\text {ion }}$ qui représente le rapport des abondances relatives des ions hydrogénoïdes (H-like) sur les ions héliumoïdes (He-like) qui sont tous les deux très majoritairement dans leur niveau fondamental. Par exemple, pour le néon, cela correspond au rapport des populations des niveaux fondamentaux $\mathrm{Ne}^{9+} / \mathrm{Ne}^{8+}$. Une forte valeur de $\mathrm{X}_{\text {ion }}$ signifie (pour un élément donné: $\mathrm{O}^{6+}$ ou $\mathrm{N}^{8+}$ ) que le nombre d'ions hydrogénoïdes est très supérieur à celui des ions héliumoïdes. Par conséquent, il y aura largement plus de recombinaisons radiatives et diélectronique (H-like $\rightarrow \mathrm{He}$-like) que 
d'excitations collisionnelles venant du niveau fondamental de l'ion héliumoïde vers les niveaux excités de la couche $n=2$ de ce même ion. L'importance des processus de recombinaison (radiative et diélectronique) est proportionnelle à $\mathrm{X}_{\text {ion }}$.

Dans le cas d'un plasma photoionisé pur, la température électronique est relativement basse, de l'ordre de $10^{4} \mathrm{~K}$ lorsqu'il s'agit des deux ions $\mathrm{O}^{6+}$ ou $\mathrm{N}^{8+}$, les processus généralement dominants de peuplement des niveaux excités sont les recombinaisons radiatives et diélectronique. Il y a lieu aussi de tenir compte du processus d'excitation collisionnelle à partir du niveau métastable $2{ }^{3} \mathrm{~S}_{1}$ vers les niveaux voisins $2{ }^{3} \mathrm{P}_{0,1,2}$ qui nécessite des énergies faibles des électrons de collision. Les niveaux $2{ }^{3} \mathrm{P}_{0,1,2}$ sont dépeuplés essentiellement par des transitions radiatives d'intercombinaison $(\mathrm{x}, \mathrm{y})$ vers le niveau fondamental $1{ }^{1} \mathrm{~S}_{0}$ et par des transitions radiatives optiquement permises vers le niveau métastable $2{ }^{3} \mathrm{~S}_{1}$. Ce dernier niveau est dépeuplé radiativement vers le niveau fondamental $1{ }^{1} \mathrm{~S}_{0}$ par une transition interdite (de type dipolaire magnétique relativiste) appelée z (voir Fig. 1). Il peut être aussi dépeuplé collisionnellement vers les trois niveaux $2{ }^{3} \mathrm{P}_{0,1,2}$. Le niveau $2{ }^{1} \mathrm{P}_{1}$ est dépeuplé radiativement vers le niveau fondamental $1{ }^{1} \mathrm{~S}_{0}$ par la transition de résonance $\mathrm{w}$.

Dans les plasmas hybrides la température électronique étant assez élevée de l'ordre de $10^{6} \mathrm{~K}$, il y a lieu d'inclure comme processus de peuplement des niveaux excités les excitations collisionnelles à partir du niveau fondamental $1{ }^{1} \mathrm{~S}_{0}$ noté 1 , vers les niveaux supérieurs, notés $2,3,4,5$ et 7 (voir figure 1).

\section{Rapport d'intensité G(Te)}

Dans cette section, nous allons établir une expression du rapport d'intensité $\mathrm{G}$ défini comme étant le rapport de la somme des intensités des raies $\mathrm{x}$, y et $z$ sur l'intensité de la raie $\mathrm{w}$, qui sont toutes émises par des ions héliumoïdes. Pour cela, il est nécessaire de déterminer les populations des niveaux supérieurs de ces trois raies. Dans un modèle collisionnel-radiatif [7] à sept niveaux incluant le niveau fondamental (noté niveau 1) et les six premiers niveaux excités de l'ion héliumoïde qui sont $1 \mathrm{~s} 2 \mathrm{~s}{ }^{3} \mathrm{~S}_{1}, 1 \mathrm{~s} 2 \mathrm{p}^{3} \mathrm{P}_{0},{ }^{3} \mathrm{P}_{1},{ }^{3} \mathrm{P}_{2}$, $1 \mathrm{~s} 2 \mathrm{~s}{ }^{1} \mathrm{~S}_{0}$ et $1 \mathrm{~s} 2 \mathrm{p}{ }^{1} \mathrm{P}_{1}$ (notés niveaux $2,3,4,5,6$ et 7 , respectivement), les équations régissant les populations de ces niveaux excités s'écrivent :

$$
\begin{aligned}
N_{2}\left(A_{21}+N_{e} \sum_{i=3}^{5} C_{2 i}\right) & =N_{1} N_{e} C_{12}+N_{3} A_{32}+N_{4} A_{42}+N_{5} A_{52}+N_{H} N_{e} C_{2}^{r} \\
N_{3} A_{32} & =N_{1} N_{e} C_{13}+N_{2} N_{e} C_{23}+N_{H} N_{e} C_{3}^{r}
\end{aligned}
$$




$$
\begin{gathered}
N_{4}\left(A_{41}+A_{42}\right)=N_{1} N_{e} C_{14}+N_{2} N_{e} C_{24}+N_{H} N_{e} C_{4}^{r} \\
N_{5}\left(A_{51}+A_{52}\right)=N_{1} N_{e} C_{15}+N_{2} N_{e} C_{25}+N_{H} N_{e} C_{5}^{r} \\
N_{7} A_{71}=N_{1} N_{e} C_{17}+N_{H} N_{e} C_{7}^{r} \\
N_{1}+N_{2}+N_{3}+N_{4}+N_{5}+N_{7}=N_{H e}
\end{gathered}
$$

où $\mathrm{N}_{H}$ désigne la densité des ions à l'état hydrogénoïde présents dans le plasma émissif (en $\mathrm{cm}^{-3}$ ), $\mathrm{N}_{\mathrm{He}}$ désigne la densité des ions héliumoïdes dans tous ses états, présents dans le plasma émissif (en $\mathrm{cm}^{-3}$ ), $\mathrm{N}_{\mathrm{e}}$ la densité électronique $\left(\mathrm{en} \mathrm{cm}^{-3}\right.$ ), et $\mathrm{C}_{\mathrm{i}}^{\mathrm{r}}$ représente le coefficient de taux de recombinaisons radiative et diélectronique (en $\mathrm{cm}^{3} \mathrm{~s}^{-1}$ ) vers les niveaux $\mathrm{i}$, et $\mathrm{C}_{\mathrm{ij}}$ représente le coefficients de taux d'excitation collisionnelle (en $\mathrm{cm}^{3} \mathrm{~s}^{-1}$ ). En écrivant ce système d'équations (5) à (10), nous avons supposé que seuls les niveaux 2 et 6 sont métastables et peuvent alors se dépeupler en partie par collisions. Cette hypothèse est largement valable tant que la densité des électrons reste inférieure à $10^{13} \mathrm{~cm}^{-3}[7]$.

Comme il a été mentionné auparavant: $\mathrm{A}_{51}<<\mathrm{A}_{52}$ et $\mathrm{A}_{42}<<\mathrm{A}_{41}$, (valable pour des numéro atomique $Z$ petit), donc les équations (5), (7) et (8) devinent :

$$
\begin{gathered}
N_{2}\left(A_{21}+N_{e} \sum_{i=3}^{5} C_{2 i}\right)=N_{1} N_{e} C_{12}+N_{3} A_{32}+N_{5} A_{52}+N_{H} N_{e} C_{2}^{r} \\
N_{4} A_{41}=N_{1} N_{e} C_{14}+N_{2} N_{e} C_{24}+N_{H} N_{e} C_{4}^{r} \\
N_{5} A_{52}=N_{1} N_{e} C_{15}+N_{2} N_{e} C_{25}+N_{H} N_{e} C_{5}^{r}
\end{gathered}
$$

Les populations $\mathrm{N}_{\mathrm{i}}$ (avec $\mathrm{i}=2-7$ ) de chaque niveau excité de l'ion héliumoïde sont négligeables devant la population $\mathrm{N}_{1}$ du niveau fondamental, ce qui entraîne que $\mathrm{N}_{\mathrm{He}} \approx \mathrm{N}_{1}$.

En substituant les deux équations (6) et (13) dans (11), on peut écrire comme équation :

$$
N_{2} A_{21}=N_{1} N_{e}\left(C_{12}+C_{13}+C_{15}\right)+N_{H} N_{e}\left(C_{2}^{r}+C_{3}^{r}+C_{5}^{r}\right)-N_{2} N_{e} C_{24}
$$

En substituant maintenant les équations (14), (12) et (9) dans l'équation (4), on obtient comme expression finale pour le rapport d'intensité $\mathrm{G}=(\mathrm{z}+\mathrm{x}+\mathrm{y}) / \mathrm{w}$ en fonction de la température électronique Te :

$$
G\left(T_{e}\right)=\frac{C_{12}+C_{13}+C_{14}+C_{15}+X_{i o n}\left(C_{2}^{r}+C_{3}^{r}+C_{5}^{r}+C_{5}^{r}\right)}{C_{17}+X_{i o n} C_{7}^{r}}
$$


Où $X_{\text {ion }}=\mathrm{N}_{\mathrm{H}} / \mathrm{N}_{1}$. On note que le rapport $\mathrm{G}$ est indépendant de la densité $\mathrm{N}_{\mathrm{e}}$. Dans la limite des basses températures, tous les coefficients de taux d'excitation $C_{1 j}(j=2-7)$ sont vraiment négligeables par rapport aux coefficients de taux de recombinaison $\mathrm{C}_{\mathrm{i}}^{\mathrm{r}}$ et G(Te) peut être approximé par :

$$
G(T e)=\frac{C_{2}^{r}+C_{3}^{r}+C_{4}^{r}+C_{5}^{r}}{C_{7}^{r}}
$$

Dans l'autre limite, celle des hautes températures, la situation est inverse, c'est-à-dire que les coefficients $C_{i}{ }^{r}$ sont très faibles devant les coefficients $C_{1 j}$, ce qui permet de faire l'approximation :

$$
G(T e)=\frac{C_{12}+C_{13}+C_{14}+C_{15}}{C_{17}}
$$

\section{Données atomiques}

Pour le calcul du rapport d'intensité $\mathrm{G}$ en fonction de la température électronique Te, les données atomiques adopter par [7].

\section{2- RESULTAS ET DISCUSSION}

Les résultats sont présentés dans les figures 2 et 3 pour les deux ions émetteurs $\mathrm{O}^{6+}$ et $\mathrm{N}^{8+}$, respectivement, où nous montrons les variations du apport d'intensité $\mathrm{G}=(\mathrm{z}+\mathrm{x}+\mathrm{y}) / \mathrm{w}$, en fonction de la température électronique Te variant dans un large domaine et en prenant le paramètre $X_{\text {ion }}=10^{\text {n }}$ avec les valeurs suivantes de $\mathrm{n}: \mathrm{n}=-1,0,1,2,3$ et 4 .

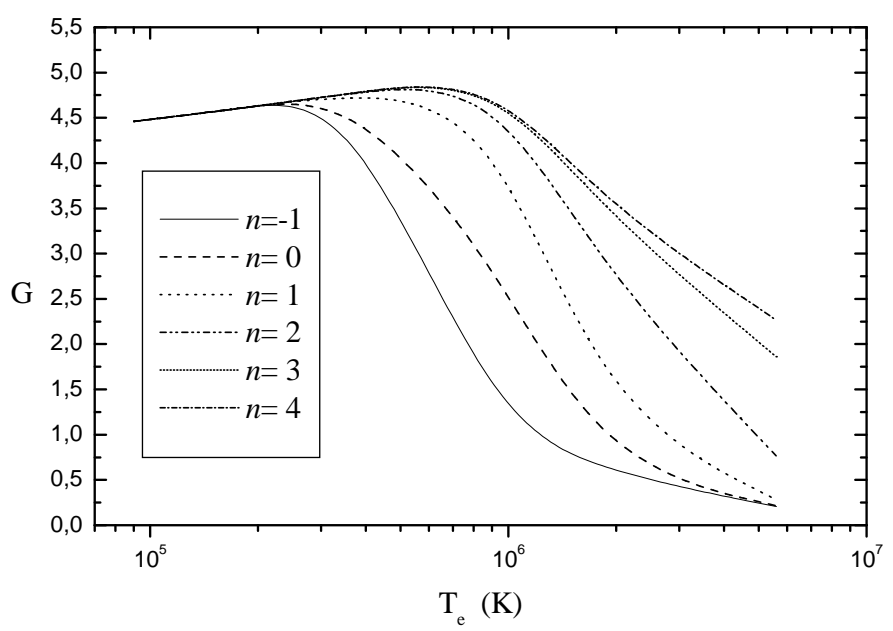

Fig.2. Variations du rapport d'intensité $G=(z+x+y) / w$ en fonction de la température 
électronique pour l'ion $\mathrm{O}^{6+}$.

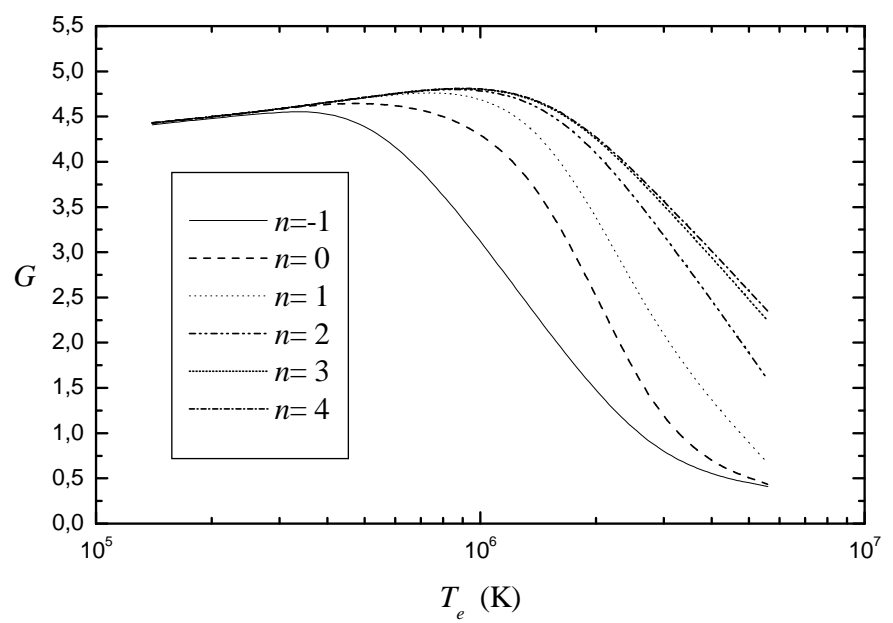

Fig.3. Variations du rapport d'intensité $\mathrm{G}=(\mathrm{z}+\mathrm{x}+\mathrm{y}) / \mathrm{w}$ en fonction de la température électronique pour l'ion $\mathrm{Ne}^{8+}$.

Dans la limite de faible température électronique tous les niveaux excités sont peuplés, par recombinaison radiative et diélectroniques, directement ou par cascade radiative venant des niveaux supérieurs (Fig.1), pour chacun des deux ions émetteurs $\mathrm{O}^{6+}$ et $\mathrm{N}^{8+}$, le rapport $\mathrm{G}$ est pratiquement indépendant de Te et en même temps de $\mathrm{X}_{\text {ion }}$ (rapport des abondances relatives des ions hydrogénoïdes sur les ions héliumoïdes), et atteint une valeur autour de 4.5. Dans ce domaine de température, le plasma est purement photoionisé et les différentes raies sont formées essentiellement par recombinaison, la température n'étant pas assez élevée pour permettre l'excitation d'un électron de la couche $n=1$ à la couche $n=2$.

A plus forte température, on voit que $G$ devient très sensible aux deux paramètres $\left(\mathrm{Te}, \mathrm{X}_{\mathrm{ion}}\right)$. Par exemple $\left(\mathrm{X}_{\text {ion }}\right.$ égal à 10 , c-à-d $\left.\mathrm{n}=1\right)$, dans le cas de $\mathrm{O}^{6+} \mathrm{G}$ décroît de la valeur 4.80 à la valeur 2.98 lorsque la température augmente de $3.6 \times 10^{5}$ à $9.0 \times 10^{5} \mathrm{~K}$ et dans le cas de $\mathrm{Ne}^{8+} \mathrm{G}$ décroît de la valeur 4.85 à la valeur 2.06 lorsque la température augmente de $1.4 \times 10^{6}$ à $2.8 \times 10^{6} \mathrm{~K}$. Pour des valeurs élevées de $\mathrm{X}_{\mathrm{ion}}$ les processus de recombinaison (radiative plus diélectronique) des ions hydrogénoïdes vers les ions héliumoïdes sont très importants du fait du très grand nombre d'ions hydrogénoïdes par rapport aux ions héliumoïdes. Dans ce cas, les raies intervenant dans G sont produites principalement par recombinaison (plasma purement photoionisé). Dans le cas contraire 
(valeurs faibles de $\mathrm{X}_{\mathrm{ion}}$ ) les raies sont produites principalement par les excitations collisionnelles (plasmas hybrides).

\section{3- CONCLUSION}

Au cours de ce travail, nous avons étudié la sensibilité en température électronique du rapport d'intensité impliquant trois raies émises dans le domaine des rayons $\mathrm{X}$ par des ions héliumoïdes de l'oxygène $\mathrm{O}^{6+}$ et néon $\mathrm{Ne}^{8+}$ présents dans des plasmas "purement photoionisés" ou "partiellement photoionisés". Le but de cette étude est son application future aux diagnostics en température électronique de certains milieux astrophysiques tels que le "Warm Absorber" (WA) existant dans des noyaux actifs de galaxies.

Les résultats que nous avons obtenus ont montré qu'il existe un domaine de valeurs de température de l'ordre de $10^{5} \mathrm{~K}$, pour lequel le rapport $\mathrm{G}$ est pratiquement indépendant de $\mathrm{Te}$ et du paramètre $\mathrm{X}_{\text {ion }}$ défini comme le rapport d'abondance relative des ions hydrogénoïdes sur les ions héliumoïdes. Dans ce domaine de température, le plasma est alors dominé par l'ionisation due aux photons. Les raies sont formées principalement par recombinaison puisque la température n'est pas assez élevée pour permettre l'excitation collisionnelle du niveau fondamental $1 \mathrm{~s}^{2}{ }^{1} \mathrm{~S}_{0}$ vers les niveaux $1 \mathrm{~s} 2 l$. Dans le domaine des hautes valeurs de température $\left(\geq 10^{6} \mathrm{~K}\right)$, le rapport $\mathrm{G}$ devient sensible aux deux paramètres $\left(\mathrm{Te}, \mathrm{X}_{\mathrm{ion}}\right)$. Pour de fortes valeurs de $\mathrm{X}_{\mathrm{ion}}$ les processus de recombinaison (radiative plus diélectronique) des ions hydrogénoïdes vers les ions héliumoïdes sont très importants du fait du très grand nombre d'ions hydrogénoïdes par rapport aux ions héliumoïdes. Dans ce cas, les raies sont produites par recombinaison (plasma purement photoionisés). Dans le cas contraire (valeurs faibles de $\mathrm{X}_{\text {ion}}$ ) les raies sont produites par les excitations collisionnelles à partir du niveau fondamental (plasmas hybrides).

\section{4- RÉFÉRENCES}

[1] Gabriel A.,H., Jordan C. it Interpretation of solar Helium-like ion line intensities. MNRAS. 1969, 145, 241-248.

[2] Pradhan A. K. Recombination-cascade X-ray spectra of highly charged helium-like ion. ApJ. 1985, 288, 824-830. 
[3] Inal M.K. and Dubau J. Theory of excitation of He-like and Li-like atomic sublevels by directive electrons: application to x-ray line polarisation. J. Phys. B : Ato. Mol. Phys. 1987, 20, 4221-4239.

[4] Kieffer J.C., Matte J.P., Chaker M., Chien C.Y., Coe S.,. Mourou G, Dubau J. and Inal M. K. J. Quant. Spectrosc. Radiat. Transfer. 1993, 48, 4648-4658.

[5] Gabriel A.,H., Jordan C. it Interpretation of spectral intensities from laboratory and astrophysical plasmas. "Case studies in atomic collision physics" .McDaniel. McDowell (eds). 1972, 2, 209.

[6] Dubau J., Volonte S. "Dielectronic recombination and its applications in astronomy ". Reports of Progress in Physics. 1980, 43, 199.

[7] Ferouani A.K (2006). Diagnostics en densité des plasmas photoionisés basés sur les raies $\mathrm{X}$ des ions héliumoïdes $\mathrm{Ne}^{8+}$. Application aux noyaux actifs de galaxies. Mémoire de Magister, 35-40 p.

[8] Bedrane Z., Inal M.K. et Fritzsche S. Effect of directional energetic electrons on the density diagnostic of hot plasmas. J. Phys. B : At. Mol. Opt. Phys. 2009, 42, 055701.

[9] Kallman T.R., McCray R. X-ray nebular models. ApJ. 1982, 50, 263.

[10] Liedahl D. A. it The X-ray spectral properties of photoinized plasmas and transient plasmas,dans "X-ray spectroscopy in Astrophysics", EADN School, Amsterdan, 1997, eds Jan van Paradjis, Johan A. M. Bleekers, 1999, 189 p.

[11] Porquet D., Dubau J. X-ray photoionized plasmas diagnostics with Helium-like ion. Application to warm absorber-emitter in active galactic nuclei. 2000, 143, 495514. 


\section{DIAGNOSTIC SPECTROSCOPIQUE EN TEMPERATURE ELECTRONIQUE DES PLASMAS PHOTOIONISES}

\section{RESUME}

Dans ce travail, nous nous intéressons au diagnostic spectroscopique en température électronique d'un plasma photoionisé, qui est basé sur le rapport d'intensité de raies appropriées émises par des ions héliumoïdes de numéro atomique $Z$ relativement petit. Comme raies appropriées, nous avons considéré les trois raies correspondant aux transitions à partir des niveaux excités $1 \mathrm{~s} 2 l$ vers le niveau fondamental $1 \mathrm{~s}^{2}{ }^{1} \mathrm{~S}_{0}$. Plus précisément, la raie de résonance $w$ due à la transition $1 \mathrm{~s} 2 \mathrm{p}^{1} \mathrm{P}_{1} \rightarrow 1 \mathrm{~s}^{2}{ }^{1} \mathrm{~S}_{0}$, la raie d'intercombinaison $(x, y) 1 \mathrm{~s} 2 \mathrm{p}{ }^{3} \mathrm{P}_{2,1} \rightarrow 1 \mathrm{~s}^{2}{ }^{1} \mathrm{~S}_{0}$ ainsi que la raie interdite $z$ due à la transition $1 \mathrm{~s} 2 \mathrm{~s}{ }^{3} \mathrm{~S}_{1} \rightarrow 1 \mathrm{~s}^{2}{ }^{1} \mathrm{~S}_{0}$. Ces raies apparaissent clairement dans les spectres des plasmas astrophysiques. Comme ion héliumoïde (He-like), nous avons choisi deux, celui l'Oxygène $\mathrm{O}^{6+}(Z=8)$ et celui le néon $\mathrm{Ne}^{8+}(Z=10)$. Nous avons effectué des calculs du rapport d'intensité $\mathrm{G}=(\mathrm{z}+\mathrm{x}+\mathrm{y}) / \mathrm{w}$ de $\mathrm{O}^{6+}$ et $\mathrm{Ne}^{8+}$ en fonction de la température électronique dans la gamme allant de $10^{5}$ à $10^{7} \mathrm{~K}$. Nous verrons que, comme il a été montré par Gabriel et Jordan en 1969 [1], ce rapport peut être très sensible à la température électronique et pratiquement indépendante de la densité électronique. Par voie de conséquence, le rapport $\mathrm{G}$ peut servir à déterminer de manière fiable la température électronique du plasma observé [2].

Mots clés : Ions fortement chargés, raies d'émission $X$, recombinaison radiative et diélectronique.

\section{How to cite this article}

Ferouani A K, Inal M K. Spectroscopic diagnosis in electronic temperature of photoionise plasmas. J Fundam Appl Sci. 2009, 1(2), 1-12. 